\title{
The study of cytoskeletal regulators on cell motility using Dictyostelium discoideum as a model organism with a view to human application in Marfan's syndrome and valvular diseases
}

\author{
Author: Gaurav Kataria
}

\section{Aims}

Several human disorders such as muscular dystrophy, Marfan's syndrome and valvular diseases have an etiology linked to filamin protein mutation. This research aimed to ascertain the role of filamin as an adaptor protein for normal cell development through exploring its influence upon cell movement and signalling.

\section{Methods}

The pre-spore cells in D. discoideum were focused upon as they produce extracellular signals (cAMP and DIF-1) involved in cell differentiation. To further investigate the role of filamin, the filamin promoter gene was firstly amplified by PCR. This was then ligated into a plasmid containing a lac $Z$ reporter gene using the restriction enzymes Bam and Eco. This was next transformed into $E$. coli for further growth and subsequently electroporated into Dictyostelium cells. Following a period of growth under optimum conditions, the cells were stained using X-Gal under the presence of a pH 7.0 Z-buffer, allowing areas of high expression to be identified. To gain insight into its influence upon cell sorting, UblA knockouts were created and the results were recorded.

\section{Results}

The results demonstrated a clear correlation between filamin expression and normal cell differentiation. They further indicated that filamin is regulated at the protein level of UblA, and that UblA downregulates a filamin binding partner. In a UblA knockout mutant, cells were seen to have a sorting defect, while in a UblA/filamin knockout, construct cells were correctly targeted to the collar region.

\section{Conclusions}

These results conclude that the role of filamin as a cytoskeletal protein does indeed have an effect upon cell movement. The relationship between UblA and filamin now allows for further research into filamin mutation disorders such as muscular dystrophies (filamin C mutation) and Marfan's syndrome/ valvular diseases (filamin A mutation). 